\title{
Optimal Control with Unreliable Communication: the TCP Case.
}

\author{
Bruno Sinopoli, Luca Schenato, Massimo Franceschetti, \\ Kameshwar Poolla, Shankar S. Sastry \\ Department of Electrical Engineering and Computer Sciences \\ University of California at Berkeley \\ $\{$ sinopoli,lusche,massimof,sastry\}@eecs.berkeley.edu \\ poolla@me.berkeley.edu
}

\begin{abstract}
The paper considers the Linear Quadratic Gaussian (LQG) optimal control problem in the discrete time setting and when data loss may occur between the sensors and the estimation-control unit and between the latter and the actuation points. We consider the case where the arrival of the control packet is acknowledged at the receiving actuator, as it happens with the common Transfer Control Protocol (TCP). We start by showing that the separation principle holds. Additionally, we can prove that the optimal LQG control is a linear function of the state. Finally, building upon our previous results on estimation with unreliable communication, the paper shows the existence of critical arrival probabilities below which the optimal controller fails to stabilize the system. This is done by providing analytic upper and and lower bounds on the cost functional.
\end{abstract}

\section{INTRODUCTION}

A growing number of applications demand remote control of plants over unreliable networks. Examples are wireless sensor networks used for estimation and control of dynamical systems [1]. In these systems issues of communication delay, data loss, and time synchronization between components play a key role. In short, communication and control become tightly coupled such that the two issues cannot be addressed independently.

Consider, for example, the problem of navigating a vehicle based on the estimate from a sensor web of its current position and velocity. The measurements underlying this estimate can be lost or delayed due to the unreliability of the wireless links. What is the amount of data loss that the control loop can tolerate to reliably perform the navigation task? Can communication protocols be designed to satisfy this constraint? The goal of this paper is to examine some control-theoretic implications of using unreliable networks for control. These require a generalization of classical control techniques that explicitly take into account the stochastic nature of the communication channel.

Communication channels typically use one of two kind of protocols: Transmission Control (TCP) or User Datagram (UDP). In the first case there is acknowledgement of received packets, while in the second case no-feedback is provided on the communication link. We study the effect of data losses due to the unreliability of the network links in the TCP case. We generalize the Linear Quadratic Gaussian

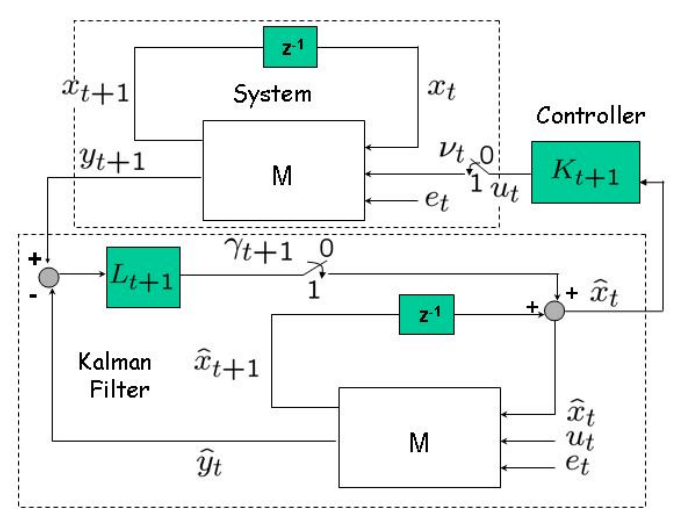

Fig. 1. Overview of the system. We study the statistical convergence of the expected state covariance of the discrete time LQG, where both the observation and the control signal, travelling over an unreliable communication channel, can be lost at each time step with probability $1-\lambda$ and $1-\nu$ respectively.

(LQG) optimal control problem - modeling the arrival of an observation as a random process whose parameters are related to the characteristics of the communication channel. Accordingly, we consider two independent Bernoulli processes, of parameters $\bar{\gamma}$ and $\bar{\nu}$, that govern packet loss between the sensors and the estimation-control unit, and between the latter and the actuation points, see Figure 1.

We first show that in the TCP case the separation principle holds and the optimal controller is a linear function of the state. We extend our previous results on estimation with missing observation packets [2] to the control case, showing the existence of critical values for the parameters of the Bernoulli arrival processes, below which a transition to instability occurs and the optimal controller fails to stabilize the system. In other words, in order to have stability, the packet loss rate must be below a given threshold that depends on the dynamics of the system.

Study of stability of dynamical systems where component are connected asynchronously via communication channels has received considerable attention in the past few years 
and our contribution can be put in the context of the previous literature. Hassibi, Zhang and Seiler [3], [4], [5]. Ling and Lemmon [6], in a series of papers, proposed a compensator approach for some data loss models. They consider an optimal compensator design when data loss is i.i.d. A different approach was considered in [7] which proposed to place an estimator, i.e. a Kalman filter, at the sensor side of the link and without assuming any statistical model for the data loss process. The work of [8] is the closest to the present paper. We consider the more general case when the matrix $C$ is not the identity and there is noise in the observation. Moreover we analyze the infinite horizon case.

Nilsson [9] presents the LQG optimal regulator with bounded delays between sensors and controller, and between the controller and the actuator, but he does not address the packet-loss case. This is considered by Hadijcostis and Touri [10], where dropped measurements are replaced by zeros. Other approaches include using the last received sample for control, or designing a dropout compensator [11], [6]. We consider the alternative approach where the external compensator feeding the controller is the optimal time varying Kalman gain. Moreover, we analyze the proposed solution in state space domain rather than in frequency domain as it was presented in [6], and we consider the more general Multiple Input Multiple Output (MIMO) case.

The separation principle states that observer and plant of a linear system can be designed independently. We first show that this principle continues to hold in the case of data loss between the sensor and the estimator. This allows us to use our result in [2], [12] to show the existence of a critical loss probability below which the resulting optimal controller fails to stabilize the system.

In our previous work on Kalman Filtering with intermittent observations [2], [12] we proved the existence of a critical loss probability under which the expected error covariance of the filter diverges. The aim of this work is to extend this result to the optimal control problem showing the existence of a transition from bounded to unbounded states in the closed loop system as well, when the rate of observation loss exceeds a given threshold $\lambda_{c}$.

Following the procedure and using the result in [2], [12] we are able to prove the existence of a critical value for the arrival rate above which the optimization problem is bounded, and below which the cost $J$ goes unbounded. This is accomplished by finding deterministic upper and lower bounds for the expected optimal cost and their convergence conditions.

The paper is organized as follows. The next section will provide a mathematical formulation for the problem. In section III we provide some preliminary results in the form of lemmas, which we then use to prove our main results in section IV. We finally conclude and provide directions for future work in section V.

\section{PROBLEM FORMULATION}

Consider the following linear stochastic system with intermittent observations:

$$
\begin{aligned}
x_{k+1} & =A x_{k}+\nu_{k} B u_{k}+w_{k} \\
y_{k} & =C x_{k}+v_{k},
\end{aligned}
$$

where $x_{k} \in \mathbb{R}^{n}$ is the state vector, $y_{k} \in \mathbb{R}^{m}$ is the output vector, $u_{k} \in \mathbb{R}^{q}$ is the input vector, $x_{0} \in \mathbb{R}^{n}, w_{k} \in \mathbb{R}^{n}$ and $v_{k} \in \mathbb{R}^{m}$ are Gaussian, uncorrelated, white, with zero mean and covariance $\left(P_{0}, Q, R_{k}\right)$ respectively, $R_{k}=\gamma_{k} R+$ $\left(1-\gamma_{k}\right) \sigma^{2} I$, and and $\left(\gamma_{k}, \nu_{k}\right)$ are i.i.d. Bernoulli random variable with $P\left(\gamma_{k}=1\right)=\bar{\gamma}$ and $P\left(\nu_{k}=1\right)=\bar{\nu}$. Let us define the following information set:

$$
\mathcal{I}_{k} \triangleq\left\{\mathbf{y}^{k}, \gamma^{k}, \nu^{k-1}\right\}
$$

where $\mathbf{y}^{k}=\left(y_{k}, y_{k-1}, \ldots, y_{1}\right), \gamma^{k}=\left(\gamma_{k}, \gamma_{k-1}, \ldots, \gamma_{1}\right)$, and $\boldsymbol{\nu}^{k}=\left(\nu_{k}, \nu_{k-1}, \ldots, \nu_{1}\right)$.

Consider also the following cost function:

$J_{N}\left(\mathbf{u}^{N-1}\right)=\mathbb{E}\left[x_{N}^{\prime} W_{N} x_{N}+\sum_{k=0}^{N-1}\left(x_{k}^{\prime} W_{k} x_{k}+\nu_{k} u_{k}^{\prime} U_{k} u_{k}\right) \mid \mathcal{I}_{N}\right]$

We now look for control input sequence $\mathbf{u}^{* N-1}$ that minimizes the above functional given that the information $\mathcal{I}_{k}$ is available at time $k$, i.e.

$$
J_{N}^{*}=\min _{\mathbf{u}^{N-1}} J_{N}\left(\mathbf{u}^{N-1}\right)=J_{N}\left(\mathbf{u}^{* N-1}\right)
$$

where $u_{k}^{*}=u_{k}^{*}\left(\mathcal{I}_{k}\right)$ and $\mathcal{I}_{k}$ is defined in Equation 3.

\section{MATHEMATICAL BACKGROUND}

Before proceeding, let us define the following variables:

$$
\begin{aligned}
\hat{x}_{k \mid k} & \triangleq \mathbb{E}\left[x_{k} \mid \mathcal{I}_{k}\right] \\
e_{k \mid k} & \triangleq x_{k}-\hat{x}_{k \mid k}, \\
P_{k \mid k} & \triangleq
\end{aligned}
$$

In the following derivation we will make use of the following facts

Lemma 1. The following facts are true:

(a) $\mathbb{E}\left[\left(x_{k}-\hat{x}_{k}\right) \hat{x}_{k}^{\prime} \mid \mathcal{I}_{k}\right]=\mathbb{E}\left[e_{k \mid k} \hat{x}_{k}^{\prime} \mid \mathcal{I}_{k}\right]=0$

(b) $\mathbb{E}\left[x_{k}^{\prime} S x_{k} \mid \mathcal{I}_{k}\right]=\hat{x}_{k}^{\prime} S \hat{x}_{k}+\operatorname{trace}\left(S P_{k \mid k}\right)=$ $\hat{x}_{k}^{\prime} S \hat{x}_{k}+\mathbb{E}\left[e_{k}^{\prime} S e_{k} \mid \mathcal{I}_{k}\right], \quad \forall S$

(c) $\mathbb{E}\left[\mathbb{E}\left[g\left(x_{k+1}\right) \mid \mathcal{I}_{k+1}\right] \mid \mathcal{I}_{k}\right]=\mathbb{E}\left[g\left(x_{k+1}\right) \mid \mathcal{I}_{k}\right], \quad \forall g(\cdot)$

Proof: (a) It follows directly from the definition. In fact: $\mathbb{E}\left[\left(x_{k}-\hat{x}_{k}\right) \hat{x}_{k}^{\prime} \mid \mathcal{I}_{k}\right]=\mathbb{E}\left[x_{k} \hat{x}_{k}^{\prime}-\hat{x}_{k} \hat{x}_{k}^{\prime} \mid \mathcal{I}_{k}\right]=$ $\mathbb{E}\left[x_{k} \mid \mathcal{I}_{k}\right] \hat{x}_{k}^{\prime}-\hat{x}_{k} \hat{x}_{k}^{\prime}=0$

(b) Using standard algebraic operations and the previous fact we have:

$$
\begin{aligned}
\mathbb{E}\left[x_{k}^{\prime} S x_{k} \mid \mathcal{I}_{k}\right] & =\mathbb{E}\left[\left(x_{k}-\hat{x}_{k}+\hat{x}_{k}\right)^{\prime} S\left(x_{k}-\hat{x}_{k}+\hat{x}_{k}\right) \mid \mathcal{I}_{k}\right] \\
& =\hat{x}_{k}^{\prime} S \hat{x}_{k}+\mathbb{E}\left[\left(x_{k}-\hat{x}_{k}\right)^{\prime} S\left(x_{k}-\hat{x}_{k}\right)\right]+ \\
& +2 \mathbb{E}\left[\hat{x}_{k}^{\prime} S\left(x_{k}-\hat{x}_{k}\right) \mid \mathcal{I}_{k}\right] \\
& =\hat{x}_{k}^{\prime} S \hat{x}_{k}+2 \operatorname{trace}\left\{S \mathbb{E}\left[\left(x_{k}-\hat{x}_{k}\right) \hat{x}_{k}^{\prime} \mid \mathcal{I}_{k}\right]\right\}+ \\
& +\operatorname{trace}\left\{S \mathbb{E}\left[\left(x_{k}-\hat{x}_{k}\right)\left(x_{k}-\hat{x}_{k}\right)^{\prime} \mid \mathcal{I}_{k}\right]\right\} \\
& =\hat{x}_{k}^{\prime} S \hat{x}_{k}+\operatorname{trace}\left\{S P_{k \mid k}\right\}
\end{aligned}
$$


(c) Let $(X, Y, Z)$ be any random vectors, $g(\cdot)$ any function, and $p$ the probability distribution, then

$$
\begin{aligned}
& \mathbb{E}_{Y, Z}[g(X, Y, Z) \mid X]= \\
& =\int_{Z} \int_{Y} g(X, Y, Z) p(Y, Z \mid X) d Y d Z \\
& =\int_{Z} \int_{Y} g(X, Y, Z) p(Y \mid Z, X) p(Z \mid X) d Y d Z \\
& =\int_{Z}\left[\int_{Y} g(X, Y, Z) p(Y \mid Z, X) d Y\right] p(Z \mid X) d Z \\
& =\mathbb{E}_{Z}\left[\mathbb{E}_{Y}[g(X, Y, Z) \mid Z, X] \mid X\right] .
\end{aligned}
$$

where we used the Bayes' Rule. Since by hypothesis $\mathcal{I}_{k} \subseteq$ $\mathcal{I}_{k+1}$, then fact (c) follows from the above equality by substituting $\mathcal{I}_{k}=X$ and $\mathcal{I}_{k+1}=(X, Z)$.

We now compute some quantities that will prove to be useful when deriving the equation for the optimal LQG controller. Let us compute the following expectation:

$$
\begin{aligned}
& \mathbb{E}\left[x_{k+1}^{\prime} S x_{k+1} \mid \mathcal{I}_{k}\right]= \\
& =\mathbb{E}\left[\left(A x_{k}+\nu_{k} B u_{k}+w_{k}\right)^{\prime} S\left(A x_{k}+\nu_{k} B u_{k}+w_{k}\right) \mid \mathcal{I}_{k}\right]= \\
& =\mathbb{E}\left[x_{k}^{\prime} A^{\prime} S A x_{k}+\nu_{k}^{2} u_{k}^{\prime} B^{\prime} S B u_{k}+w_{k}^{\prime} S w_{k}+\right. \\
& \left.+2 \nu_{k} u_{k}^{\prime} B^{\prime} S A x_{k}+2\left(A x_{k}+\nu_{k} B u_{k}\right) w_{k} \mid \mathcal{I}_{k}\right]= \\
& =\mathbb{E}\left[x_{k}^{\prime} A^{\prime} S A x_{k} \mid \mathcal{I}_{k}\right]+\bar{\nu} u_{k}^{\prime} B^{\prime} S B u_{k}+ \\
& +2 \bar{\nu} u_{k}^{\prime} B^{\prime} S A \mathbb{E}\left[x_{k} \mid \mathcal{I}_{k}\right]+\operatorname{trace}\left(S \mathbb{E}\left[w_{k} w_{k}^{\prime} \mid \mathcal{I}_{k}\right]\right)= \\
& =\mathbb{E}\left[x_{k}^{\prime} A^{\prime} S A x_{k} \mid \mathcal{I}_{k}\right]+\bar{\nu} u_{k}^{\prime} B^{\prime} S B u_{k}+ \\
& +2 \bar{\nu} u_{k}^{\prime} B^{\prime} S A \hat{x}_{k \mid k}+\operatorname{trace}(S Q)
\end{aligned}
$$

where we used independence of $\nu_{k}, w_{k}, x_{k}$, and zero-mean property of $w_{k}$. The previous expectation hold true for both the information sets $\mathcal{I}_{k}=\left\{\mathcal{I}_{k}, \mathcal{G}_{k}\right\}$. Also

$\mathbb{E}\left[e_{k \mid k}^{\prime} T e_{k \mid k} \mid \mathcal{I}_{k}\right]=\operatorname{trace}\left(T \mathbb{E}\left[e_{k \mid k} e_{k \mid k}^{\prime} \mid \mathcal{I}_{k}\right]\right)=\operatorname{trace}\left(T P_{k \mid k}\right)$

\section{Finite AND InfInite HoRIZON LQG}

We first start finding the optimal estimator, which will be needed to solve the LQG controller design, as it will be shown later.

\section{A. Estimator Design, $\sigma \rightarrow+\infty$}

We derive the equations for optimal estimator using similar arguments used for the standard Kalman filtering equations. The innovation step is given by:

$$
\begin{aligned}
\hat{x}_{k+1 \mid k} \triangleq & \mathbb{E}\left[x_{k+1} \mid \nu_{k}, \mathcal{I}_{k}\right]=\mathbb{E}\left[A x_{k}+\nu_{k} B u_{k}+w_{k} \mid \nu_{k}, \mathcal{I}_{k}\right] \\
= & A \mathbb{E}\left[x_{k} \mid \mathcal{I}_{k}\right]+\nu_{k} B u_{k} \\
& =A \hat{x}_{k \mid k}+\nu_{k} B u_{k} \\
e_{k+1 \mid k} \triangleq & x_{k+1}-\hat{x}_{k+1 \mid k} \\
= & A x_{k}+\nu_{k} B u_{k}+w_{k}-\left(A \hat{x}+\nu_{k} B u_{k}\right) \\
= & A e_{k \mid k}+w_{k} \\
& P_{k+1 \mid k} \triangleq \mathbb{E}\left[e_{k+1 \mid k} e_{k+1 \mid k}^{\prime} \mid \nu_{k}, \mathcal{I}_{k}\right]= \\
& =\mathbb{E}\left[\left(A e_{k \mid k}+w_{k}\right)\left(A e_{k \mid k}+w_{k}\right)^{\prime} \mid \nu_{k} \mathcal{I}_{k}\right] \\
& =A \mathbb{E}\left[e_{k \mid k} e_{k \mid k}^{\prime} \mid \mathcal{I}_{k}\right] A^{\prime}+\mathbb{E}\left[w_{k} w_{k}^{\prime}\right] \\
& =A P_{k \mid k} A^{\prime}+Q
\end{aligned}
$$

where we used the independence of $w_{k}$ and $\mathcal{I}_{k}$. Since $y_{k+1}, \gamma_{k+1}, w_{k}$ and $\mathcal{I}_{k}$ are all independent of each other and following the same approach described in [2], then correction step is given by:

$$
\begin{aligned}
\hat{x}_{k+1 \mid k+1} & =\hat{x}_{k+1 \mid k}+\gamma_{k+1} K_{k+1}\left(y_{k+1}-C \hat{x}_{k+1 \mid k}\right) \\
e_{k+1 \mid k+1} & =x_{k+1}-\hat{x}_{k+1 \mid k+1} \\
& =x_{k+1}-\left(\hat{x}_{k+1 \mid k}+\gamma_{k+1} K_{t+1}\left(y_{k+1}-C \hat{x}_{k+1 \mid k}\right)\right. \\
& =e_{k+1 \mid k}-\gamma_{k+1} K_{t+1}\left(C x_{t+1}+v_{k+1}-C \hat{x}_{k+1 \mid k}\right) \\
& =\left(I-\gamma k+1 K_{k+1} C\right) e_{k+1 \mid k}-\gamma_{k+1} K_{k+1} v_{k+1}(14) \\
P_{k+1 \mid k+1} & =P_{k+1 \mid k}-\gamma_{k+1} K_{k+1} C P_{k+1 \mid k}, \\
K_{k+1} & \triangleq P_{k+1 \mid k} C^{\prime}\left(C P_{k+1 \mid k} C^{\prime}+R\right)^{-1}
\end{aligned}
$$

where we took the limit $\sigma \rightarrow+\infty$.

The initial conditions for the estimator iterative equations are:

$$
\begin{aligned}
& \hat{x}_{0 \mid-1}=0 \\
& P_{0 \mid-1}=P_{0}
\end{aligned}
$$

\section{B. Controller design}

To derive the optimal feedback control law and the corresponding value for the objective function we will follow the dynamic programming approach based on the cost-to-go iterative procedure.

Define the optimal value function $V_{k}\left(x_{k}\right)$ as follows:

$$
\begin{gathered}
V_{N}\left(x_{N}\right) \triangleq \mathbb{E}\left[x_{N}^{\prime} W_{N} x_{N} \mid \mathcal{I}_{N}\right] \\
V_{k}\left(x_{k}\right) \triangleq \min _{u_{k}} \mathbb{E}\left[x_{k}^{\prime} W_{k} x_{k}+\nu_{k} u_{k}^{\prime} U_{k} u_{k}+V_{k+1}\left(x_{k+1}\right) \mid \mathcal{I}_{k}\right]
\end{gathered}
$$

Using dynamic programming theory [13] [14], one can show that $J_{N}^{*}=V_{0}\left(x_{0}\right)$.

We claim that the value function $V_{k}\left(x_{k}\right)$ can be written as:

$$
V_{k}\left(x_{k}\right)=\mathbb{E}\left[x_{k}^{\prime} S_{k} x_{k} \mid \mathcal{F}_{k}\right]+c_{k}, \quad k=0, \ldots, N
$$

where the matrix $S_{k}$ and the scalar $c_{k}$ are to be determined and are independent of the information set $\mathcal{I}$. We will prove it by induction. The claim is certainly true for $k=N$ with the following choice of parameters:

$$
\begin{aligned}
S_{N} & =W_{N} \\
c_{N} & =0
\end{aligned}
$$

Suppose now that the claim is true for $k+1$, i.e. $V_{k+1}\left(x_{k+1}\right)=\mathbb{E}\left[x_{k+1}^{\prime} S_{k+1} x_{k+1} \mid \mathcal{F}_{k+1}\right]+c_{k+1}$, and we use it to compute the value function at time step $k$ as follows: 


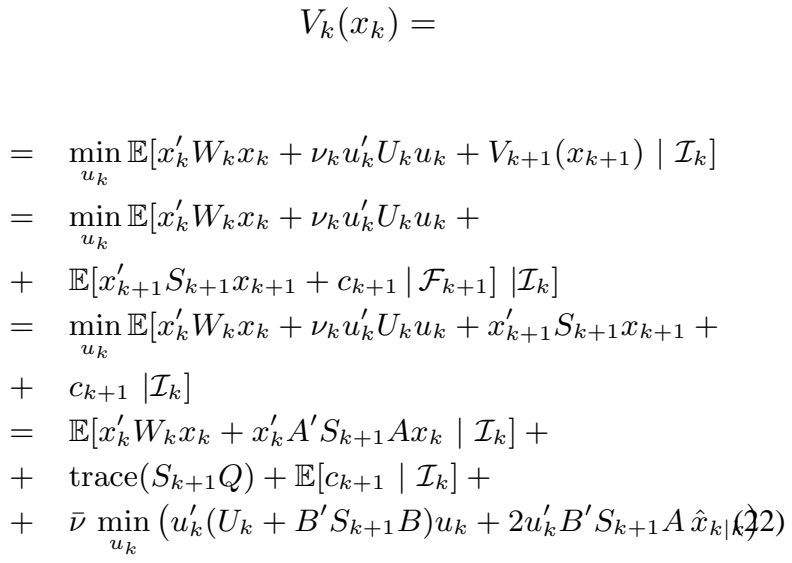

where we used Lemma 1(c) in the third line, and Equation (7) in the last two lines. The value function is a quadratic function of the input, therefore the minimizer can be simply obtained by solving $\frac{\partial V_{k}}{\partial u_{k}}=0$, which gives:

$$
u_{k}=-\left(B^{\prime} S_{k+1} B+U_{k}\right)^{-1} B^{\prime} S_{k+1} A \hat{x}_{k \mid k}=L_{k} \hat{x}_{k \mid k} .
$$

The optimal feedback is thus a simple linear feedback of the estimated state. If we substitute the minimizer back into Equation (22), and we use the Equation (19) we get:

$$
\begin{aligned}
& V_{k}\left(x_{k}\right)= \\
& =\mathbb{E}\left[x_{k}^{\prime} W_{k} x_{k}+x_{k}^{\prime} A^{\prime} S_{k+1} A x_{k} \mid \mathcal{I}_{k}\right]+\operatorname{trace}\left(S_{k+1} Q\right)+ \\
& +\mathbb{E}\left[c_{k+1} \mid \mathcal{I}_{k}\right]-\bar{\nu} \hat{x}_{k \mid k}^{\prime} A^{\prime} S_{k+1} B\left(U_{k}+B^{\prime} S_{k+1} B\right)^{-1} B^{\prime} S_{k+1} A \hat{x}_{k \mid k} \\
& \mathbb{E}\left[x_{k}^{\prime} S_{k} x_{k} \mid \mathcal{I}_{k}\right]+c_{k}= \\
& =\mathbb{E}\left[x_{k}^{\prime} W_{k} x_{k}+x_{k}^{\prime} A^{\prime} S_{k+1} A x_{k}-\right. \\
& \left.+\bar{\nu} x_{k}^{\prime} A^{\prime} S_{k+1} B\left(U_{k}+B^{\prime} S_{k+1} B\right)^{-1} B^{\prime} S_{k+1} A x_{k} \mid \mathcal{I}_{k}\right]+ \\
& +\operatorname{trace}\left(S_{k+1} Q\right)+\mathbb{E}\left[c_{k+1} \mid \mathcal{I}_{k}\right]+ \\
& +\bar{\nu} \operatorname{trace}\left(A^{\prime} S_{k+1} B\left(U_{k}+B^{\prime} S_{k+1} B\right)^{-1} B^{\prime} S_{k+1} P_{k \mid k}\right)(25)
\end{aligned}
$$$$
\widetilde{P}_{k \mid k} \leq \mathbb{E}_{\gamma}\left[P_{k \mid k}\right] \leq \widehat{P}_{k \mid k}
$$$$
\begin{aligned}
J_{N}^{*} & =V_{0}\left(x_{0}\right)=\mathbb{E}\left[x_{0}^{\prime} S_{0} x_{0}\right]+ \\
& +\sum_{k=0}^{N-1}\left(\operatorname{trace}\left(\left(A^{\prime} S_{k+1} A+W_{k}-S_{k}\right) \mathbb{E}_{\gamma}\left[P_{k \mid k}\right]\right)+\right. \\
& \left.+\operatorname{trace}\left(S_{k+1} Q\right)\right) \\
& =\bar{x}_{0}^{\prime} S_{0} \bar{x}_{0}+\operatorname{trace}\left(S_{0} P_{0}\right)+ \\
& +\sum_{k=0}^{N-1}\left(\operatorname{trace}\left(\left(A^{\prime} S_{k+1} A+W_{k}-S_{k}\right) \mathbb{E}_{\gamma}\left[P_{k \mid k}\right]\right)+\right. \\
& \left.+\operatorname{trace}\left(S_{k+1} Q\right)\right)
\end{aligned}
$$

The matrices $\left\{P_{k \mid k}\right\}_{k=0}^{N}$ are stochastic since they are function of the sequence $\left\{\gamma_{k}\right\}$. The exact expected value of these matrices cannot be computed analytically, since they are nonlinear function of the arrival sequence $\gamma_{k}$, as shown in [2]. However, they can bounded by computable determiniatic quantities. In fact let us consider the following equation:

$$
\begin{aligned}
\widehat{P}_{k+1 \mid k} & =A \widehat{P}_{k \mid k-1} A^{\prime}+Q- \\
& +\bar{\gamma} A \widehat{P}_{k \mid k-1} C^{\prime}\left(C \widehat{P}_{k \mid k-1} C^{\prime}+R\right)^{-1} C \widehat{P}_{k \mid k-1} A^{\prime} \\
\widehat{P}_{k \mid k} & =\widehat{P}_{k \mid k-1}-\bar{\gamma} \widehat{P}_{k \mid k-1} C^{\prime}\left(C \widehat{P}_{k \mid k-1} C^{\prime}+R\right)^{-1} C \widehat{P}_{k \mid} \\
\widetilde{P}_{k+1 \mid k} & =(1-\bar{\gamma}) A \widetilde{P}_{k \mid k-1} A^{\prime}+Q \\
\widetilde{P}_{k \mid k} & =(1-\bar{\gamma}) \widetilde{P}_{k \mid k-1}
\end{aligned}
$$

initialized to $\widehat{P}_{0 \mid-1}=\widetilde{P}_{0 \mid-1}=P_{0}$. Using similar arguments as those in [2], it is possible to show that the matrices $P_{k \mid k}$ 's are concave and monotonic functions of $P_{k \mid k-1}$, respectively. Therefore, the following bounds are true:

where we used Lemma 1(b) in the last line. For the previous equation to hold for all $x_{k}$, we need to have:

$$
\begin{aligned}
S_{k} & =A^{\prime} S_{k+1} A+W_{k}- \\
& +\bar{\nu} A^{\prime} S_{k+1} B\left(B^{\prime} S_{k+1} B+U_{k}\right)^{-1} B^{\prime} S_{k+1} A \\
c_{k} & =\bar{\nu} \operatorname{trace}\left(A^{\prime} S_{k+1} B\left(U_{k}+B^{\prime} S_{k+1} B\right)^{-1} B^{\prime} S_{k+1} P_{k \mid k}\right)+ \\
& +\operatorname{trace}\left(S_{k+1} Q\right)+\mathbb{E}\left[c_{k+1} \mid \mathcal{I}_{k}\right] \\
& =\operatorname{trace}\left(\left(A^{\prime} S_{k+1} A+W_{k}-S_{k}\right) P_{k \mid k}\right)+ \\
& +\operatorname{trace}\left(S_{k+1} Q\right)+\mathbb{E}\left[c_{k+1} \mid \mathcal{I}_{k}\right]
\end{aligned}
$$

Therefore, the cost function for the optimal LQG using
Therefore we have:

\section{Finite and Infinite Horizon LQG control}

The previous equations were derived for the finite horizon LQG. The infinite horizon LQG can be obtained by taking the limit for $N \rightarrow+\infty$ of the previous equations. However, the matrices $\left\{M_{k+1}\right\}$ and $\left\{P_{k \mid k}\right\}$ depend on the specific realization of the observation sequence $\left\{\gamma_{k}\right\}$, therefore the minimal cost $J_{N}$ is a stochastic function and does not have a limit. Differently from standard LQG controller design

$$
\begin{aligned}
J_{N}^{\min } & \leq J_{N}^{*} \leq J_{N}^{\max } \\
J_{N}^{\max } & =\bar{x}_{0}^{\prime} S_{0} \bar{x}_{0}+\operatorname{trace}\left(S_{0} P_{0}\right)+ \\
& +\sum_{k=0}^{N-1}\left(\operatorname{trace}\left(\left(A^{\prime} S_{k+1} A+W_{k}-S_{k}\right) \widehat{P}_{k \mid k}\right)+\right. \\
& \left.+\operatorname{trace}\left(S_{k+1} Q\right)\right) \\
J_{N}^{\min } & =\bar{x}_{0}^{\prime} S_{0} \bar{x}_{0}+\operatorname{trace}\left(S_{0} P_{0}\right)+ \\
& +\sum_{k=0}^{N-1}\left(\operatorname{trace}\left(\left(A^{\prime} S_{k+1} A+W_{k}-S_{k}\right) \widetilde{P}_{k \mid k}\right)\right. \\
& \left.+\operatorname{trace}\left(S_{k+1} Q\right)\right)
\end{aligned}
$$


where the controller always stabilizes the original system, in the case control packet loss, the stability can be lost if the arrival probability $\bar{\nu}, \bar{\gamma}$ is below a certain threshold. In particular the Equation for the cost matrix $S_{k}$ is the solution of a modified Riccati Algebraic Equation (MARE) which was already introduced and studied in our previous work [2]. In particular, Equation (26) is the dual of the estimator equation presented in [2]. Therefore, the same conclusions can be drown and we are now ready summarize the previous result in the following theorem:

Theorem 1 (Finite Horizon LQG under TCP). Consider the system (1)-(2) and consider the problem of minimizing the cost function (4) with policy $u_{k}=f\left(\mathcal{I}_{k}\right)$, where $\mathcal{I}_{k}$ is the information available under TCP communication, given in Equation (3). Then, the optimal control is a linear function of the estimated system state given by Equation (23), where the matrix $S_{k}$ can be computed iteratively using Equation (26). The separation principle still hold under TCP communication, since the optimal estimator is independent of the control input $u_{k}$. The optimal state estimator is given by Equations (9)-(13) and (12)-(16), and the minimal achievable cost is given given by Equation (28).

Theorem 2 (Infinite Horizon LQG under TCP). Consider the same systems as defined in the previous theorem with the following additional hypothesis: $W_{N}=W_{k}=W$ and $U_{k}=U$. Moreover, let $(A, B)$ and $\left(A, Q^{\frac{1}{2}}\right)$ be controllable, and let $(A, C)$ and $\left(A, W^{\frac{1}{2}}\right)$ be observable. Let us consider the limiting case $N \rightarrow+\infty$, then, there exist arrival probabilities $\nu_{\min }$ and $\gamma_{\min }$ which satisfy the following property:

$$
\begin{aligned}
& \min \left(1,1-\frac{1}{\left|\lambda_{\max }(A)\right|^{2}}\right) \leq \nu_{\min } \leq 1, \\
& \min \left(1,1-\frac{1}{\left|\lambda_{\max }(A)\right|^{2}}\right) \leq \gamma_{\min } \leq 1,
\end{aligned}
$$

where $\left|\lambda_{\max }(A)\right|$ is the eigenvalue of matrix $A$ with largest absolute value, such that for all $\bar{\gamma}>\gamma_{\min }$ we have:

$$
\begin{aligned}
& L_{k}=L_{\infty}=-\left(B^{\prime} S_{\infty} B+U\right)^{-1} B^{\prime} S_{\infty} A \\
& \frac{1}{N} J_{N}^{m i n} \leq \frac{1}{N} J_{N}^{*} \leq \frac{1}{N} J_{N}^{\max }
\end{aligned}
$$

where the mean cost bounds $J_{N}^{\min }, J_{N}^{\max }$ are given by:

$$
\begin{aligned}
J_{\infty}^{\max } & =\lim _{N \rightarrow+\infty} \frac{1}{N} J_{N}^{\max } \\
& =\operatorname{trace}\left(( A ^ { \prime } S _ { \infty } A + W _ { k } - S _ { \infty } ) \left(\widehat{P}_{\infty}-\right.\right. \\
& \left.\left.+\widehat{\gamma}_{\infty} C^{\prime}\left(C \widehat{P}_{\infty} C^{\prime}+R\right)^{-1} C \widehat{P}_{\infty}\right)\right)+\operatorname{trace}\left(S_{\infty} Q\right) \\
J_{\infty}^{\text {min }} & =\lim _{N \rightarrow+\infty} \frac{1}{N} J_{N}^{\text {min }} \\
& =(1-\bar{\gamma}) \operatorname{trace}\left(\left(A^{\prime} S_{\infty} A+W_{k}-S_{\infty}\right) \widetilde{P}_{\infty}\right)+ \\
& +\operatorname{trace}\left(S_{\infty} Q\right)
\end{aligned}
$$

and the matrices $S_{\infty}, \bar{P}_{\infty}, \underline{P}_{\infty}$

$S_{\infty}=A^{\prime} S_{\infty} A+W-\bar{\nu} A^{\prime} S_{\infty} B\left(B^{\prime} S_{\infty} B+U\right)^{-1} B^{\prime} S_{\infty} A$

$\bar{P}_{\infty}=A \bar{P}_{\infty} A^{\prime}+Q-\bar{\gamma} A \bar{P}_{\infty} C^{\prime}\left(C \bar{P}_{\infty} C^{\prime}+R\right)^{-1} C \bar{P}_{\infty} A^{\prime}$

$\underline{P}_{\infty}=(1-\bar{\gamma}) A \underline{P}_{\infty} A^{\prime}+Q$

Moreover, the assumptions above are necessary and sufficient conditions for boundedness of cost function under $L Q G$ feedback. The critical probabilities $\gamma_{\min }$ and $\nu_{\min }$ can be computed via the solution of the following LMIs optimization problems:

$$
\begin{aligned}
& \gamma_{\min }=\operatorname{argmin}_{\bar{\gamma}} \Psi_{\gamma}(Y, Z)>0, \quad 0 \leq Y \leq I . \\
& \Psi_{\gamma}(Y, Z)= \\
& =\left[\begin{array}{ccc}
Y & \sqrt{\gamma}(Y A+Z C) & \sqrt{1-\gamma} Y A \\
\sqrt{\gamma}\left(A^{\prime} Y+C^{\prime} Z^{\prime}\right) & Y & 0 \\
\sqrt{1-\gamma} A^{\prime} Y & 0 & Y
\end{array}\right] \\
& \nu_{\min }=\operatorname{argmin}_{\bar{\nu}} \Psi_{\nu}(Y, Z)>0, \quad 0 \leq Y \leq I . \\
& =\left[\begin{array}{cc}
Y, Z)= \\
\sqrt{\nu}\left(A Y+B Z^{\prime}\right) \\
\sqrt{1-\nu} A Y \\
\mathrm{~V} . \text { ConCLUSION }
\end{array}\right]
\end{aligned}
$$

\section{CONCLUSION}

Motivated by applications where control is performed over a communication network, in this paper we extend our previous results on optimal control with intermittent observations to the case where control packets may be lost due to the presence of an unreliable communication channel between the controller and the actuator. We assume that an acknowledgement of the arrival of the control packet is always available to the controller (TCP). First, we showed that the separation principle holds also in this case. Then we proved that the optimal LQG control is a linear function of the state. Finally, by providing analytic upper and and lower bounds on the cost functional we could show the existence of critical arrival probabilities below which the optimal controller fails to stabilize the system. Future work will involve the analysis for the case when the controller does not receive any acknowledgement to whether its packet has been received by the actuator or not.

\section{REFERENCES}

[1] B. Sinopoli, C. Sharp, S. Schaffert, L. Schenato, and S. Sastry, "Distributed control applications within sensor networks," IEEE Proceedings Special Issue on Distributed Sensor Networks, November 2003.

[2] B. Sinopoli, L. Schenato, M. Franceschetti, K. Poolla, M. Jordan, and S. Sastry, "Kalman filtering with intermittent observations," IEEE Transactions on Automatic Control, September 2004, to appear.

[3] A. Hassibi, S. Boyd, and J. How, "Control of asynchronous dynamical systems with rate constraints on events," in Proceedings of the 38th IEEE Conference on Decision and Control, Phoenix, AZ, December 1999.

[4] W. Zhang, M. S. Branicky, and S. M. Philips, "Stability of networked control systems," IEEE Control System Magazine, vol. 21(1), pp. 8489, February 2001. 
[5] P. Seiler, "Coordinated control of unmanned aerial vehicles," Ph.D. dissertation, University of California at Berkeley, Berkeley, CA, 2001.

[6] Q. Ling and M. Lemmon, "Optimal dropout compensation in networked control systems," in IEEE conference on decision and control, December 2003, maui, HI.

[7] V. Gupta, D. Spanos, B. Hassibi, and R. M. Murray, "Optimal lqg control across a packet-dropping link," California Institute of technology, Tech. Rep., 2004, preprint, submitted for publication.

[8] O. C. Imer, S. Yuksel, and T. Basar, "Optimal control of dynamical systems over unreliable communication links," in NOLCOS, Stutgart, Germany, 2004.

[9] J. Nilsson, "Real-time control systems with delays," Ph.D. dissertation, Department of Automatic Control, Lund Institute of Technology, 1998.

[10] C. N. Hadjicostis and R. Touri, "Feedback control utilizing packet dropping network links," in Proceedings of the 41st IEEE Conference on Decision and Control, Las Vegas, NV, Dec 2002, invited.

[11] Q. Ling and M. Lemmon, "Soft real-time scheduling of networked control systems with dropouts governed by a markov chain," in American Control Conference, June 2003, denver, CO.

[12] B. Sinopoli, L. Schenato, M. Franceschetti, K. Poolla, M. Jordan, and S. Sastry, "Kalman filtering with intermittent observations," in IEEE Conference on Decision and Control, December 2003.

[13] P. Kumar and P. Varaiya, Stochastic Systems: Estimation, Identification and Adaptive Control, ser. Information and System Science Series, T. Kailath, Ed. Englewood Cliffs, NJ 07632: Prentice Hall, 1986.

[14] D. Bertsekas and J. Tsitsiklis, Neuro-Dynamic Programming. Athena Scientific, September 1996. 